\title{
A location optimization method for aircraft weakly-rigid structures
}

\author{
Zhong-Qi Wang, Yuan Yang*, Yong-Gang Kang, and Zheng-Ping Chang \\ The Key Laboratory of Contemporary Design and Integrated Manufacturing Technology, Northwestern Polytechnical University, \\ 710072 Xi'an, Shaanxi, P.R. China
}

Received 23 June 2013 / Accepted 23 October 2013 / Published online 26 February 2014

\begin{abstract}
Since aircraft weakly-rigid structure has large size and weak stiffness, there has serious deformation during assembly process. The current deformation analysis theory of rigid assembly is not applicable. Based on the $N-2-1$ $(N>3)$ locating principle, this paper presents a methodology for weakly-rigid parts. An optimization algorithm combines finite element analysis and nonlinear programming methods to find the optimal number and position of the locating points in order to minimize the assembly deformation. An example application study is presented to demonstrate the optimization procedure and its effectiveness by using the software of ABAQUS.
\end{abstract}

Key words: Weakly-rigid structures, $N$-2-1 Locating principle, Location optimization, ABAQUS.

\section{Introduction}

By virtue of high strength, large deflection, and light weight, weakly-rigid structures are widely used in the aerospace industry, as important parts of fuselage, wings, horizontal stabilizer, and vertical stabilizer. Different from rigid structures, they always tend to distort and deform during assembly, which causes the assembly deformation $[1,2]$. The problems may adversely affect the final product functionality and the process performance. For this reason, many domestic and foreign scholars and technicians have carried out the research [3-7]. More research concentrates on the auto body assembly positioning, while little on weakly-rigid for aircraft assembly. This paper focuses on the impact of locators (number and position) on the dimensional quality. The locator layout is based on an $\mathrm{N}$-2-1 locating principle, which has more than three locators in the primary plane. The optimization approach combines finite element analysis and nonlinear programming methods in finding the optimal number of locators as well as their positions that minimizes assembly deformation.

\section{Optimization algorithm of locating points}

As described above, it is better to use the $N-2-1(N>3)$ locating principle than the 3-2-1 scheme for weakly-rigid assemblies to avoid the assembly deformation and assure the assembly quality. Figure 1 shows a typical $N-2-1$ locating principle $(N=6)$.

For weakly-rigid structures assembly, there is a set of points on components, called key measurement points (KMPs), which are utilized to evaluate the assembly dimensional quality [8, 9]. In this study, we choose the boundary points and midpoints as KMPs. Optimization objective function is set to the deformation caused by gravity at KMPs, and it is convenient and rapid to calculate the deformation using ABAQUS. According to required precision, a proper mesh generation should be performed on the component. Then calculate the deformation with finite element method. Residual strain and work hardening of weakly-rigid component caused by metal stamping is not considered in this study.

\subsection{Objective function and constraints}

Suppose that there are $L$ number of KMPs. The deformation of KMPs in $X$ - and $Y$-axes of part coordinate under the action of dead load is so small that we assume its location in $X$ - and $Y$-axes is in an ideal position. So the paper focuses on the deformation in Z-axis:

$$
\operatorname{Minimize} F(\vec{X}),=\sum_{i}^{L} U_{i}(\vec{X})^{2},
$$

$$
\text { Subject to } G_{1}(\vec{X})=0 \text {, }
$$

*e-mail: yangyuan0824@mail.nwpu.edu.cn 


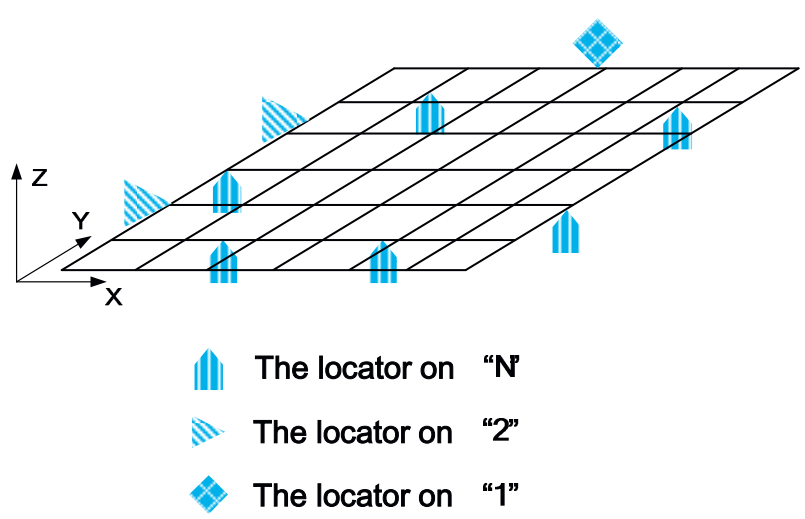

Figure 1. N-2-1 Locating principle of weakly-rigid structures.

$$
G_{2}(\vec{X}) \geq 0
$$

where $\vec{X}$ is a vector which stands for $N$ number of design variables or the position of locating points. Therefore, equation (1) can be rewritten as:

$$
\begin{aligned}
\min F(\vec{X}) & =\sum_{i}^{L} U_{i}(\vec{X})^{2} \\
& =F\left(V_{1}, \ldots, V_{j}, V_{j+1}, \ldots, V_{N}\right),
\end{aligned}
$$

where $j=1,2, \ldots, N, V_{j}$ stands for the position of the $j$-th locating point.

$F(\vec{X})$ is the optimization objective function. $L$ is the number of KMPs, and the deformation of the KMPs is considered in $F(\vec{X}) . U_{i}(\vec{X})$ stands for normal displacement of the $i$-th KMP. $G_{1}(\vec{X})$ and $G_{2}(\vec{X})$ denote respectively equality constraints and inequality constraints of design variables, that is, the region in which the locators can exist. $F(\vec{X})$ is a nonlinear function of $\vec{X}$, while $G_{1}(\vec{X})$ and $G_{2}(\vec{X})$ are linear functions. Considering local deformation, the deformation displacement of KMPs should subject to constraint condition as follows:

$$
\left|U_{i}(\vec{X})\right| \leq \Omega \text {. }
$$

where $\Omega$ is the allowed maximum displacement for each locating point.

\subsection{Optimization method}

From the mathematical model, it is seen that the non-linear objective function is like a "black-box" and its properties are unknown [10]. Aiming at this optimization problem, the procedure can be described with the following steps:

1. Perform a proper mesh generation on the component according to the principle of mesh generation, and set a couple of supporting points at certain intervals in the feasible area of the component. Then number the points 1 , $2, \ldots, N$.

2. Calculate $F(\vec{X})$ under gravity, if $F(\vec{X})$ is too large, it should adjust the spacing of the supporting points, so that $F(\vec{X})$ is controlled within a certain range;

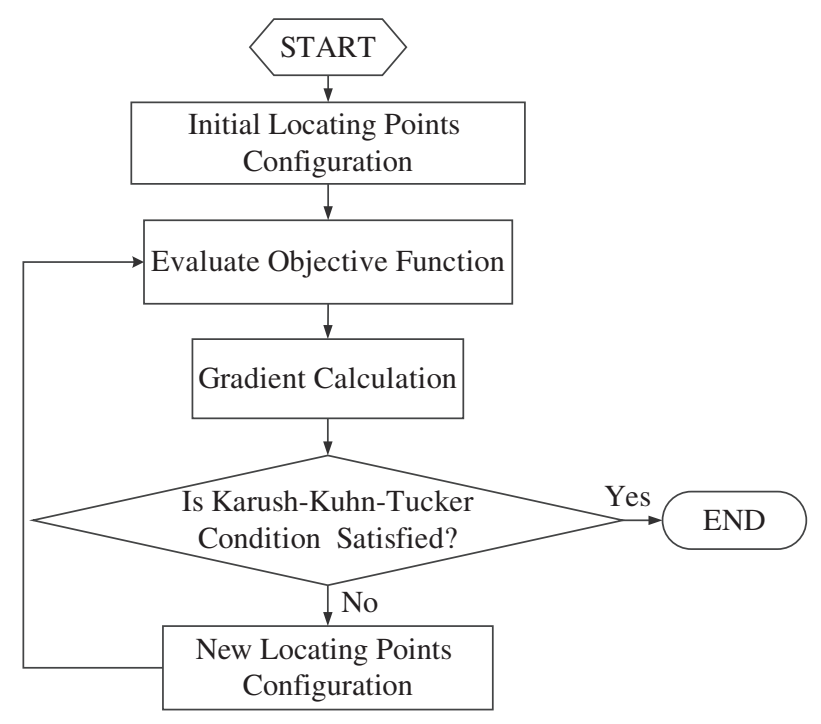

Figure 2. Flow chart of the optimization method.

Table 1. The parameters of the material.

\begin{tabular}{lc}
\hline Parameter & Value \\
\hline Mass density & $6.98 \times 10^{3} \mathrm{~kg} / \mathrm{m}^{3}$ \\
Young's modules & $2 \times 10^{5} \mathrm{MPa}$ \\
Poisson ratio & 0.23 \\
\hline
\end{tabular}

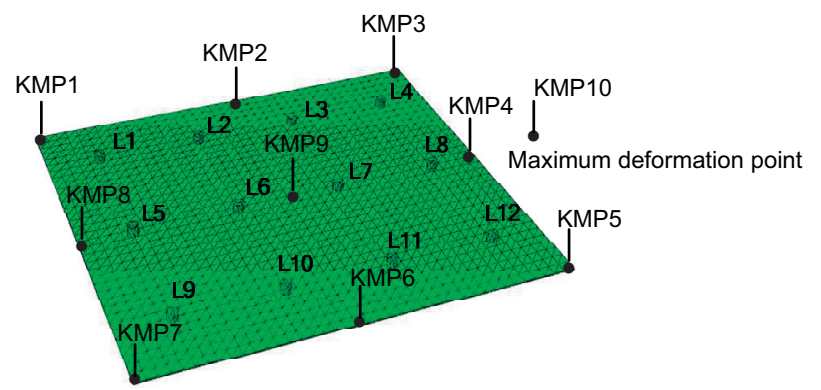

Figure 3. Mesh generation of sheet metal part.

3. Sequentially remove the supporting points $1,2,3, \ldots, N$, calculate $F(\vec{X})$ when each supporting point was removed, and find the point which has the minimal impact on the component and take it away. Now, there are $(N-1)$ number of supporting points left.

4. Among the $(N-1)$ supporting points, remove each one sequentially, then search the point having minimal impact on the component and take it away. Now the number of the supporting points is reduced to $(N-2)$.

5. Repeat step 4 until a certain stopping criterion is met.

Due to the lack of a close form function for $F(\vec{X})$, the objective function gradient vector $g$ can be approximated using finite difference method:

$$
g_{i}=\frac{F\left(X+\Delta X_{i}\right)-F(X)}{\Delta X_{i}} .
$$




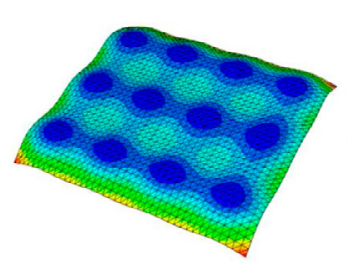

$N=12$

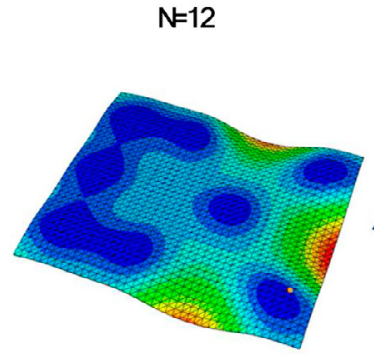

$N=8$

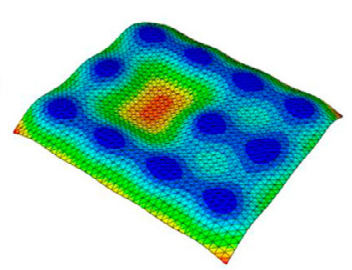

$N=11$

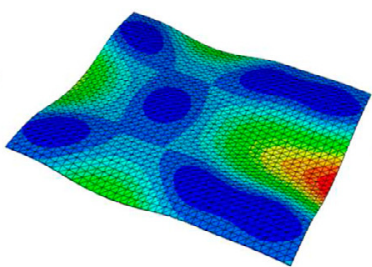

$N=7$

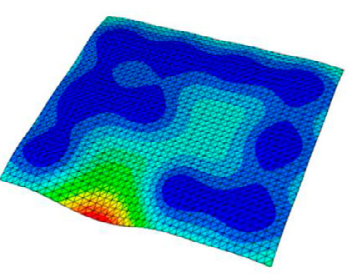

$N=10$

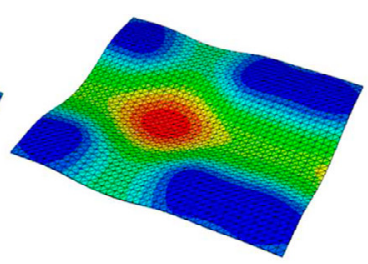

$N=6$

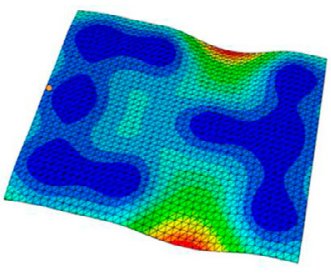

$\mathrm{N}=9$

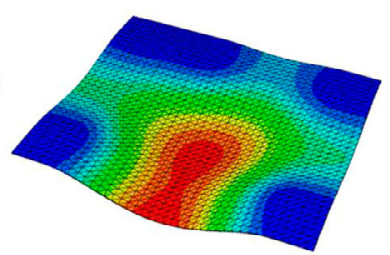

$N=5$

Figure 4. Result comparison in the software of ABAQUS.

Table 2. Objective function values when $N=5,6,7\left(\times 10^{-3} \mathrm{~mm}\right)$.

\begin{tabular}{lccc}
\hline Number of KMPs & \multicolumn{3}{c}{ Absolute value of displacement } \\
\cline { 2 - 4 } & $N=5$ & $N=6$ & $N=7$ \\
\hline KMP1 & 2.44 & 2.31 & 2.04 \\
KMP2 & 4.46 & 5.19 & 5.7 \\
KMP3 & 2.48 & 2.42 & 2.45 \\
KMP4 & 13 & 14.2 & 13.3 \\
KMP5 & 1.4 & 2.44 & 2.43 \\
KMP6 & 36.2 & 5.09 & 5.63 \\
KMP7 & 1.47 & 2.32 & 2 \\
KMP8 & 11.9 & 12.8 & 8.97 \\
KMP9 & 30.9 & 17.8 & 2.39 \\
$\Sigma$ & 2611.9765 & 757.6927 & 347.3189 \\
KMP10 & 36.2 & 19.2 & 13.3 \\
\hline
\end{tabular}

where $\Delta X_{i}$, defined as $\Delta X_{i}=\left[0, \ldots, \delta_{i}, \ldots, 0\right]^{T}$, is a perturbation vector of the design variable $\vec{X}$ in the $i$-direction. Now, the number and position of positioning points is preferred. To sum up, the whole optimization process can be illustrated in Figure 2.

\section{Application example}

\subsection{Problem description}

In this section, the optimization methodology is illustrated by a sheet metal part. It has dimensions $400 \times 350 \times 1.5 \mathrm{~mm}^{3}$. The parameters of the material can be shown as Table 1. Generally speaking, the KMPs need to be determined according to the demand of practical production, and in this paper, we choose the boundary points and midpoints as the KMPs. In order to be more representative, the point having the maximum deformation is also chosen as one of the indicators to measure parts deformation, we call it KMP10. The configuration of 10 KMPs is shown as Figure 3. Now, we have $(9+1)$ number of KMPs and $(3 \times 4)$ number of initial locating points.
The constraint condition of locating point $\left(x_{j}, y_{j}\right)$ :

$$
\begin{aligned}
& 0 \leq x_{j} \leq 400 j=1,2, \ldots, 12 . \\
& 0 \leq y_{j} \leq 350 j=1,2, \ldots, 12 .
\end{aligned}
$$

The constraint condition of the deformation in normal direction of each KMP:

$$
\left|U_{i}(\vec{X})\right| \leq \Omega=0.02 \mathrm{~mm} i=1,2, \ldots, L,(L=9) .
$$

The finite element method result in this study only considers the gravity of the part, but in some real situations, other constraint conditions during assembly should be taken into consideration, such as the deformation caused by clamps, etc. And we must take them into consideration in the finite element analysis, if the deformation has a large effect on the sheet metal part.

\subsection{Optimization result}

After having removed the point having the minimal impact on the part in turn, the deformation cloud diagram in ABAQUS $(N=12,11,10,9,8,7,6,5)$ is shown in Figure 4 .

Table 2 shows the deformation displacement and its square sum when $N=5,6$ and 7 respectively. It can be seen, when it has five locators, there are three KMPs (KMP6, KMP9 and KMP10) whose deformation displacement exceeds the set maximum allowable displacement. When it has six locators, the deformation and its square sum of each KMP are decreased significantly, and the set accuracy is met. As the number of locators is increased to 7 , with the increment of the number of locators, the deformation displacement of each KMP still has a downward trend. But considering the cost of clamping equipment and the increment of coupling error may caused by too 
many over-positioning, select the "6-2-1" locator layout scheme in the case study is reasonable. At the same time, the example also verified the "3-2-1" positioning principle is difficult to meet the positioning requirements for weakly-rigid structures.

\section{Conclusions}

For the $N-2-1(N>3)$ locating principle of the weakly-rigid structures, the location performance not only depends on the number, but also the position of the locators. In this paper, combining finite element analysis and nonlinear programming methods, an optimization method is proposed. With the using of finite element software of ABAQUS, the optimal number of locators as well as their positions can be found. Furthermore, a case study also illustrated the effectiveness and practicability of the proposed method.

Acknowledgements. This work is supported by National Natural Science Foundation of China (Grant No. 51375396).

\section{References}

1. Cheng H, Li Y, Zhang KF, Su JB. 2011. Efficient method of positioning error analysis for aeronautical thin-walled structures multi-state riveting. International Journal of Advanced Manufacturing Technology, 55, 217-233.
2. Liao XY, Wang GG. 2008. Simultaneous optimization of fixture and joint positions for non-rigid sheet metal assembly. International Journal of Advanced Manufacturing Technology, 36, 386-394

3. Rearick MR, Hu SJ, Wu SM. 1993. Optimal fixture design for deformable sheet metal workpieces. Transactions of NAMRI/ SME, 11, 407-412.

4. Cai W, Hu SJ, Yuan JX. 1996. Deformable sheet metal fixturing: principles, algorithms, and simulations. ASME Journal of Manufacturing Science and Engineering, 118(3), 318-324.

5. Camelio J, Hu SJ, Ceglarek D. 2004. Impact of fixture design on sheet metal assembly variation. Journal of Manufacturing Systems, 23(3), 182-193.

6. Luo LJ. 2002. Auto body compliant assembly welding fixture design method research based on weld feature and variation control. Shanghai Jiao Tong University: Shanghai.

7. Zhu JH, Zhang WH. 2006. Maximization of structural natural frequency with optimal support layout. Structural and Multidisciplinary Optimization, 31, 462-469.

8. Xing YF. 2008. Assembly sequence optimization of auto-body parts based on compliant assembly variation analysis. Shanghai Jiao Tong University: Shanghai.

9. Ruan YM, Liu YX. 2009. A thin plate welding fixture point layout optimization method. HEAVY TRUCK, 3, 22-24.

10. Li KH. 2007. Study on Application of Designed Method of Automotive Welding Jig by CATIA. Chongqing University: Chongqing.

Cite this article as: Wang QZ, Yang GY, Kang GY \& Chang ZP: A location optimization method for aircraft weakly-rigid structures. Int. J. Simul. Multisci. Des. Optim., 2014, 5, A18. 